\title{
Application value of conventional ultrasound combined with shear wave elastography in diagnosing triple negative breast cancer
}

\author{
Changrui Sheng, Shanshan Gao, Liming Yan, Hua Yin, Jingjing Hu, Zhiying Ye, Xiuzhi Wei \\ Department of Ultrasound, Hwa Mei Hospital, University of Chinese Academy of Sciences, Ningbo, China \\ Contributions: (I) Conception and design: C Sheng, S Gao; (II) Administrative support: L Yan; (III) Provision of study materials or patients: C Sheng, \\ S Gao, H Yin; (IV) Collection and assembly of data: J Hu, Z Ye, X Wei; (V) Data analysis and interpretation: J Hu, Z Ye, X Wei; (VI) Manuscript \\ writing: All authors; (VII) Final approval of manuscript: All authors. \\ Correspondence to: Shanshan Gao. Department of Ultrasound, Hwa Mei Hospital, University of Chinese Academy of Sciences, 41 Xibei Road, Ningbo \\ 315010, China. Email: ritagao1981@163.com.
}

Background At present, most ultrasound (US) studies on triple negative breast cancer (TNBC) are limited to conventional US features, so it is necessary to develop new joint diagnostic methods. The study aims to explore the values of conventional US and shear wave elastography (SWE) in differential diagnoses of TNBC and non-TNBC.

Methods: A total of 120 breast cancer (BC) patients involving 120 lesions that were pathologically verified were retrospectively analyzed in this study. All participants had received both conventional US and SWE before surgery. Meanwhile, the participants were divided into a TNBC group or a non-TNBC group according to their immunohistochemical (IHC) results. The differences between the conventional US features (including lesion shape, growth location, margin, boundary, internal echo, micro-calcification, posterior echo, and internal blood supply) and the SWE image features [including mean lesion hardness (Emean), maximum (Emax), minimum (Emin), standard deviation (SD), and ratio to normal gland (Eratio)] of 2 groups were compared. The receiver operating characteristic (ROC) curve of the diagnosed lesion was calculated by the area under the curve (AUC).

Results: According to the findings of conventional US, the TNBC group mostly manifested as a microlobulated margin, with a clear boundary and no internal micro-calcification; the non-TNBC group mainly manifested as marginal angulation or burr, and hyper-echo halo in the boundary, accompanied with internal micro-calcification, and the difference was statistically significant $(\mathrm{P}<0.05)$; the internal thrombolysis in myocardial infarction (TIMI) and resistance index between the TNBC group and non-TNBC group were similar, and the differences were not statistically significant $(\mathrm{P}>0.05)$. The findings of SWE were as follows: differences in Emax, Emean, and Eratio values between 2 groups were statistically significant $(\mathrm{P}<0.05)$; and the areas under the ROC curve (AUC) of these three in diagnosing the lesions were $0.811,0.781$ and 0.770 , respectively.

Conclusions: Conventional US combined with SWE can comprehensively analyze the morphological, blood supply, and hardness features of breast lesions, and provide more reliable information for the differential diagnosis between TNBC and non-TNBC.

Keywords: Ultrasonography; shear wave elastography (SWE); triple negative breast cancer (TNBC)

Submitted Mar 24, 2021. Accepted for publication Jun 18, 2021.

doi: $10.21037 /$ gs-21-320

View this article at: https://dx.doi.org/10.21037/gs-21-320 


\section{Introduction}

In recent years, the morbidity of breast cancer (BC) shows an increasing annual trend, which has become the malignant tumor with the highest morbidity among Chinese women $(1,2)$. Triple negative BC (TNBC) is a BC subtype with negative estrogen receptor (ER), progesterone receptor (PR) and human epidermal growth factor receptor (HER2) (3-5), which accounts for $10-17 \%$ of all BC cases (6). It is associated with high invasion, frequent local recurrence and distant metastasis (DM), as well as poor prognosis (7). Morphologically, TNBC is likely to display benign tumor features $(2,8,9)$, which increases the likelihood of it being misdiagnosed as a benign lesion, thus delaying patient treatment. Therefore, it is of particular importance to diagnose TNBC early. At present, there have been extensive studies on TNBC oncology and pathology, as well as studies on TNBC in terms of imaging, magnetic resonance imaging (MRI), and X-ray photography, but few ultrasonic studies on TNBC have been reported. However, there are some shortcomings to ultrasonic diagnosis. Ultrasound diagnostic capability is impaired by associated benign features encountered in $21-41 \%$ of TNBC lesions (10). Triple-negative breast cancer lacks the typical malignant US features of breast cancer (11).The TNBC manifestations of 2-dimensional (2D) ultrasound (US) and US elastography have particular characteristics, which have significance in distinguishing TNBC from non-TNBC, but color Doppler flow imaging (CDFI) shows that the blood flow signals of TNBC and non-TNBC are similar. There are few reports on the differential diagnosis of TNBC and non-TNBC by contrast-enhanced US and 3D ultrasound. There are still some challenges to the early diagnosis of TNBC and the differential diagnosis between TNBC and other types of BC.

Shear wave elastography (SWE) is a novel elastography pattern. The shear waves generated by acoustic radiation forces propagate transversely through the tissue and the speed was rescored. It can measure and reflect the tissue elasticity value, quantitatively assess the hardness of lesions, and provide objective data for diagnosis. In the report (12), SWE has been prospectively confirmed that it could reflect lymphedema even at a latent stage for patients with breast cancer after surgery because of the fibrotic soft tissue change in stiffness. Our study aimed to explore the application of SWE's advantage in measuring tissue hardness combined with SWE in the differential diagnosis of TNBC and non-TNBC, thus enhancing the imaging foundation for preoperative diagnosis of TNBC.

We present the following article in accordance with the STARD reporting checklist (available at https://dx.doi. org/10.21037/gs-21-320).

\section{Methods}

\section{Objects of study}

A total of $120 \mathrm{BC}$ patients involving 120 lesions that were pathologically verified through surgery in our hospital from September 2016 to December 2017 were retrospectively analyzed. All participants were female aged 23-79 years old, with the average age $(45.32 \pm 14.29)$ years old. The inclusion criteria were as follows: (I) patients received bilateral breast US before surgery; (II) core needle biopsy and surgical treatment were carried out after US; (III) postoperative specimens were subjected to pathological examination and immunohistochemical (IHC) detection; (IV) patients with complete US, pathological, and IHC data. The exclusion criteria were as follows: (I) patients had undergone chemotherapy or radiotherapy before surgery; (II) patients had not received SWE before surgery; (III) the preoperatively preserved US images were of poor quality; (IV) the greatest diameter of breast lesion was $\geq 8 \mathrm{~cm}$; (V) multiple or diffuse BC.

\section{Instrument and methods}

\section{Instrument}

The Supersonic Imagine Aixplorer diasonograph (SuperSonic Imagine S.A., Aix-en-Provence, France) was adopted, with the L15-4 linear array probe at the frequency of 4-15 MHz.

\section{Ultrasonography}

Images were collected by 2 physicians with over 5 years' experience of breast US diagnosis, who then underwent SWE technical training, as shown below: (I) patients assumed the supine position; the bilateral breasts were sufficiently exposed; conventional US was carried out to determine the lesion position; multi-dimension and multisection scanning was performed, and the complete lesion gray scale US image information was preserved. (II) The detection pattern was switched to CDFI, sampling frame size was adjusted, probe was slowly moved from one side of the lesion to the other side, and the images were preserved; in addition, the spectral Doppler waveform features were 
obtained, arterial resistance index was measured, and the data were recorded. (III) The probe was switched to the SWE pattern without applying pressure, sampling frame was selected (to cover the entire lesion as much as possible), the patient was asked to hold their breath for 5-10 s, frame was stopped at 3-4 s after the image had stabilized, and the images were then captured. The sampling frame diameter in the region of interest (ROI) was $2 \mathrm{~mm}$, then the ROI was placed in the position with the greatest tumor hardness. Later, the mean (Emean), maximum (Emax), minimum (Emin), and standard deviation (SD) of the Young's modulus, together with the ratio of Emean of disease tissue to normal gland tissue (Eratio), were measured; the section with maximum diameter and the corresponding vertical section of the same lesion were selected, the same ROI was positioned and measured repeatedly in triplicate; finally, 3 groups of data were obtained, including the Emean, Emax, Emin, SD, and Eratio values of the lesion. Upon measurement, the images were preserved, and the mean of each index was calculated.

\section{Parameter analysis}

The parameters were analyzed by 2 sonographers with certain clinical diagnostic experience, and the analysis criteria were as follows: (I) according to the standards in the second version of the Breast Imaging Reporting and Data System (BI-RADS, 2013) by the American College of Radiology (13), the 7 features of mass on conventional 2D US were assessed, including mass shape (round/oval or irregular), growth location (aspect ratio $>1$ or aspect ratio $<1$ ), margin (smooth, micro-tubular, angulation/ spur), boundary (hyper-echo halo, clear), internal echo (hypoecho, mixed echo, extreme hypoecho), microcalcification (yes/no), posterior echo feature (unchanged, enhanced, or attenuated); (II) the blood flow was assessed according to the Alder semi-quantitative classification (14), grades 0 -III were divided into 0 -I grade and II-III grade for comparison; and the difference in blood flow resistance index was compared. (III) The Emean, Emax, Emin, SD, and Eratio ratios measured by SWE were compared for their differences. The above analyses were performed by 2 physicians, and any disagreement between them was addressed through mutual discussion to reach a consensus.

Determination of ER, PR, and HER-2 results are as follows: pathological examination and IHC detection were performed by pathologists. The BC tissues were subjected to paraffin embedding, sectioning, and observation using the IHC Envision method. As stipulated in the Breast
Cancer Diagnosis and Treatment Guideline and Standard [2017] by the Chinese Anti-Cancer Association (15), ER and PR expression of 0 was judged negative, while + , ++ and +++ were deemed positive; HER-2 of - or + was deemed negative, while that of +++ was regarded positive; for participants with ++ HER-2, fluorescence in situ hybridization (FISH) was performed to detect whether the HER-2 gene was amplified, those with amplification were deemed positive, while those with no amplification were regarded as negative. All BC which were ER, PR, and HER-2 were defined as TNBC, and if any one of them or multiple indexes were positive, they were defined as nonTNBC.

All procedures performed in this study involving human participants were in accordance with the Declaration of Helsinki (as revised in 2013). This study was approved and supervised by the Research Ethics Committee of the Hwa Mei Hospital (No. YJ-KYSB-NBEY-2018-055-01). Informed consent was provided by all participants.

\section{Statistical analysis}

The statistical software SPSS version 24.0 (SPSS Inc., IBM Corp., Armonk, NY, USA) was used for statistical analysis. Enumeration data were expressed as $\mathrm{n}(\%)$ and compared by chi-square test between 2 groups; measurement data were expressed as $\bar{x} \pm \mathrm{s}$, and those conforming to normal distribution with homogeneity of variance were compared using $t$-test in inter-group comparison. A $\mathrm{P}$ value $<0.05$ was deemed statistically significant. The curve regarding the Young's modulus values was plotted with IHC analysis results as the gold standard, the area under the receiver operating characteristic (ROC) curve (AUC) was calculated, the optimal diagnosis cutoff value was selected based on the highest critical point of Youden index (sensitivity + specificity - 1), and the corresponding sensitivity and specificity were calculated.

\section{Results}

\section{Clinicopathological features}

A total of 120 breast lesions were enrolled in this study: (I) $42(35.0 \%)$ cases were in the TNBC group, with the maximum diameter of $0.62-4.24 \mathrm{~cm}$, and the average of $(1.62 \pm 0.65) \mathrm{cm}$, among them, $33(78.57 \%)$ cases were invasive ductal carcinomas, $5(11.90 \%)$ were intraductal carcinomas, and 4 (9.53\%) were invasive lobular carcinomas; 

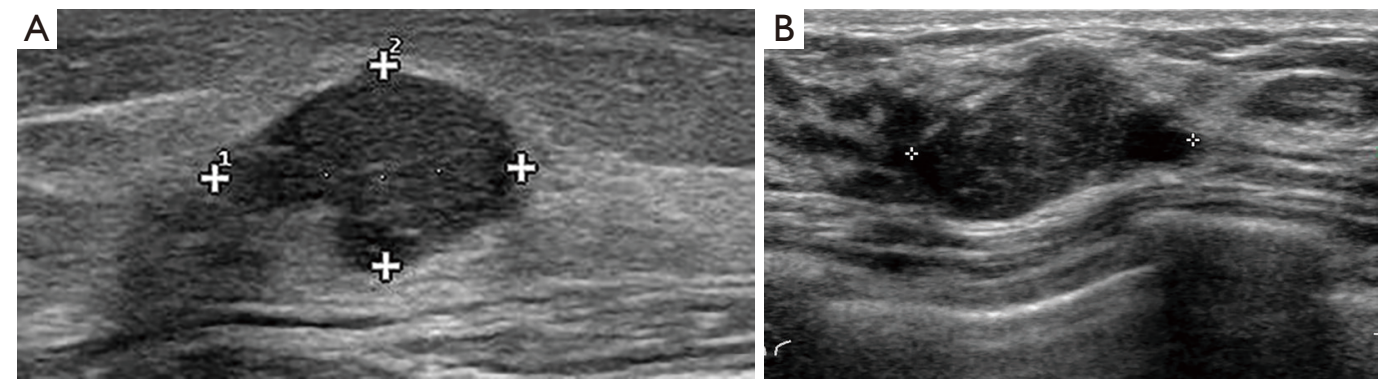

Figure 1 Conventional ultrasound findings between TNBC and non-TNBC patients. (A) Lesion margin is micro-lobular in shape, with clear boundary, and no internal micro-calcification; IHC results: TNBC; (B) angulation and spur in lesion margin, with hyper echo halo in the surrounding, and internal micro-calcification, IHC results: non-TNBC. TNBC, triple negative breast cancer; IHC, immunohistochemical.

(II) there were $78(65.0 \%)$ cases in the non-TNBC group, the maximum diameter was $0.52-6.02 \mathrm{~cm}$, with an average of $(21.37 \pm 1.05) \mathrm{cm}$, among them, $63(80.77 \%)$ were invasive ductal carcinomas, $8(10.26 \%)$ were intraductal carcinomas, and $7(8.97 \%)$ were invasive lobular carcinomas.

\section{Conventional US findings}

Comparisons of conventional US findings: (I) TNBC mostly manifested as a micro-lobular margin $(57.1 \%$, $24 / 42)$, with clear boundary $(83.3 \%, 35 / 42)$ and with no internal micro-calcification $(63.3 \%, 27 / 42)$ (Figure 1A); (II) in contrast, the non-TNBC was mainly manifested as spur and angulation in the margin $(56.4 \%, 44 / 78)$, high echo halo in the boundary $(65.4 \%, 51 / 78)$, and internal microcalcification (66.7\%, 52/78) (Figure 1B); (III) compared with non-TNBC, the shape of TNBC mass was mostly regular (38.1\% vs. $14.1 \%, \mathrm{P}<0.05)$, and the posterior echo was mostly enhanced (28.6\% vs. $7.7 \%, \mathrm{P}<0.05)$ (Table 1 ).

Comparisons of Doppler US findings: the thrombolysis in myocardial infarction (TIMI) and resistance index in lesion of TNBC group showed no statistically significant difference compared with those in the non-TNBC group $(\mathrm{P}>0.05)$ (Figure 2; Table 1).

Comparisons of SWE parameters and ROC curve analysis: (I) the Emean, Emax, and Eratio values in the TNBC group were remarkably lower than those in the non-TNBC group, and the differences were statistically significant $(\mathrm{P}<0.05)$ (Figure 3, Table 2); (II) differences in Emin and SD between the 2 groups were not statistically significant $(\mathrm{P}>0.05)$ (Table 2).

The ROC curve was plotted with sensitivity as the vertical coordinate and1-specificity as the horizontal coordinate; the AUC of Emean, Emax, and Eratio were $0.781,0.811$, and 0.770 , respectively (Figure 4), and the optimal cutoff values were $77.8 \mathrm{kPa}$ (sensitivity of $55.6 \%$, specificity of $96.2 \%$ ), $127.0 \mathrm{kPa}$ (sensitivity of $79.1 \%$, specificity of $71.4 \%$ ), and $8.7 \mathrm{kPa}$ (sensitivity of $72.7 \%$, specificity of $76.0 \%$ ), respectively.

\section{Discussion}

It is common for TNBC to occur in relatively young premenopausal women, which is associated with poor cell differentiation, high invasion, and high early recurrence risk within 1-3 years following diagnosis $(16,17)$; besides, a majority of TNBC cases die within the first 5 years after treatment. However, neoadjuvant chemotherapy has led to a high pathological remission rate for TNBC $(15,18,19)$, and it is of particular importance for the early diagnosis and early treatment of TNBC. At present, the US diagnosis of TNBC is mostly restricted to conventional US and the traditional assisted elastography. Previous research has indicated that conventional US is likely to confuse TNBC with benign lesions, and the difference in the hardness between TNBC and non-TNBC is not statistically significant $(20,21)$. As a novel elastography pattern, SWE requires no application of manual external force and can thereby avoid the influence of the operator and (or) tissue on the tissue strain; concurrently, it can quantitatively evaluate lesion hardness, and provide more objective diagnostic information, which is superior to the assisted elastography semi-quantitative evaluation method. Consequently, SWE has provided a novel technical approach to distinguish whether there is a difference in the hardness between TNBC and non-TNBC. 
Table 1 Comparison of ultrasound characteristics between TNBC and non-TNBC

\begin{tabular}{|c|c|c|c|c|c|c|c|c|c|c|c|}
\hline Group & Number & \multicolumn{2}{|c|}{ Shape } & \multicolumn{2}{|c|}{ Growth orientation } & \multicolumn{3}{|c|}{ Internal echo } & \multicolumn{3}{|c|}{ Edge } \\
\hline TNBC & 42 & 16 & 26 & 26 & 16 & 22 & 9 & 11 & 6 & 24 & 12 \\
\hline Non-TNBC & 78 & 11 & 67 & 35 & 43 & 55 & 6 & 17 & 9 & 25 & 44 \\
\hline$\chi^{2}$ & & \multicolumn{2}{|c|}{9.012} & \multicolumn{2}{|c|}{3.169} & \multicolumn{3}{|c|}{5.747} & \multicolumn{3}{|c|}{8.908} \\
\hline \multirow{2}{*}{ Group } & \multicolumn{2}{|c|}{ Boundary } & \multicolumn{2}{|c|}{ Microcalcification } & \multicolumn{4}{|c|}{ Posterior echo } & \multicolumn{2}{|c|}{ Blood flow } & Resistance \\
\hline & Clear & Unclear & Yes & No & Unchanged & Enhe & ance Atte & nuation & 0 -I stage & II-IV stage & index \\
\hline TNBC & 36 & 7 & 27 & 15 & 21 & 1 & 2 & 9 & 25 & 17 & $0.73 \pm 0.08$ \\
\hline$P$ value & \multicolumn{2}{|c|}{0.00} & \multicolumn{2}{|c|}{0.003} & \multicolumn{4}{|c|}{0.044} & \multicolumn{2}{|c|}{0.26} & 0.876 \\
\hline
\end{tabular}

TNBC, triple negative breast cancer.
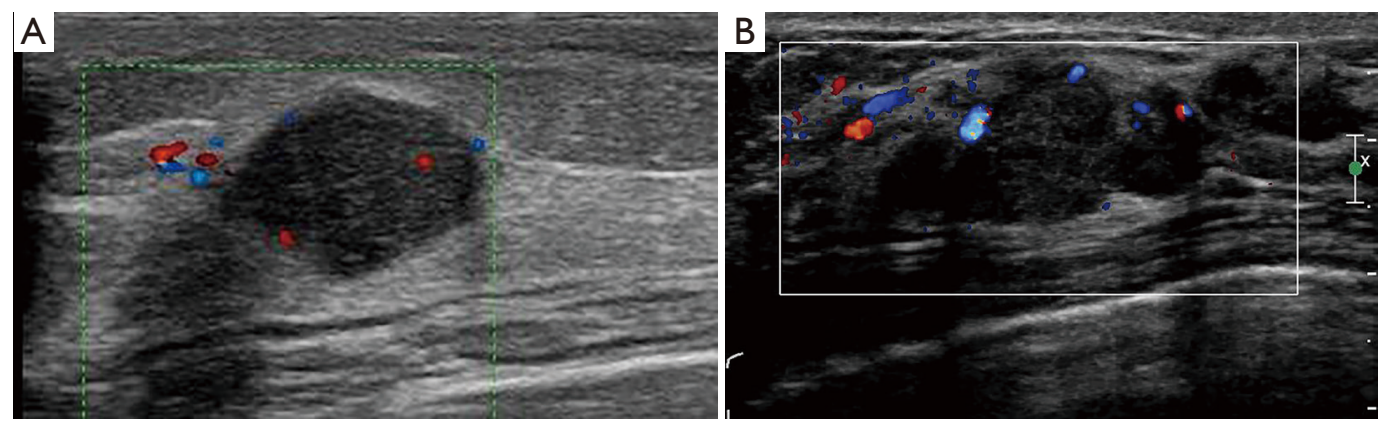

Figure 2 Color Doppler ultrasonography findings between TNBC and non-TNBC patients. (A) Lesion Alder TIMI of grade II, IHC results: TNBC; (B) lesion Alder TIMI of grade II, IHC results: non-TNBC. TNBC, triple negative breast cancer; IHC, immunohistochemical; TIMI, thrombolysis in myocardial infarction; Red and blue dot, blood flow signals.

Our research results demonstrated that, in conventional US, the TNBC lesion mainly manifested as micro-lobular margin, with clear boundary, regular shape, and mostly posterior echo enhancement, but with no internal microcalcification, which was in line with the latest research results in China and internationally in the past few years $(2,5,16,22,23)$. The TNBC lesion had micro-lobular margin, with clear boundary and regular shape, which might be related to the growth pattern of TNBC tumor cells. As suggested in previous studies, TNBC tumor cells grow at a fast speed, display "squeezing" or "expansive" growth, and will compress the surrounding tissues, with clear boundaries. Contrastingly, non-TNBC tumor cells exhibit "invasive" growth, which invades the surrounding tissues; these cancer cells mix with fats and fibrous tissues, accompanied by responsive hyperplasia of surrounding connective tissues, leading to the appearance of an irregular interface; it manifests as irregular shape, margin angulation or spur, and the formation of a hyper-echoic halo in the boundary on ultrasonogram. Our results indicated that, compared with non-TNBC, posterior echo enhancement was more common in TNBC, which was consistent with the research results of Kojima et al. (24) and $\mathrm{Li}$ et al. (25). This might have been related to the fact that a large number of tumor cells and lymphocytes had gathered in the TNBC lesion, which frequently resulted in central necrosis, 

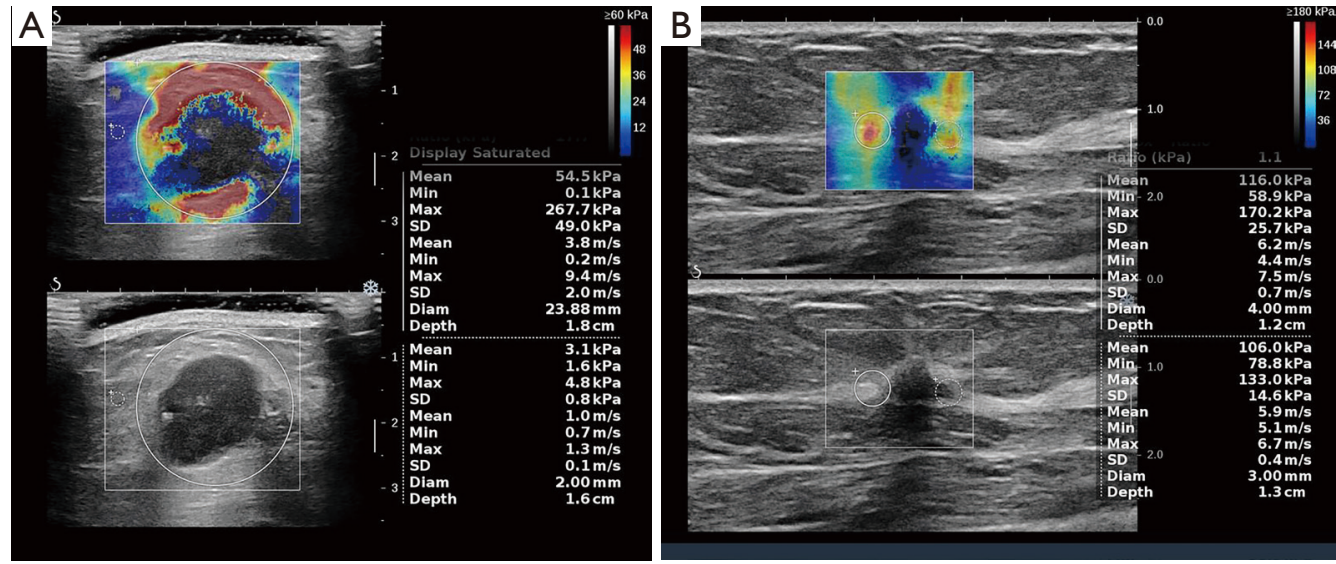

Figure $3 \mathrm{SWE}$ images of TNBC and non-TNBC patients. (A) TNBC, Emean =56.2 kPa, Emax =78.8 $\mathrm{kPa}$, Eratio =2.6; (B) non-TNBC, Emean $=97.9 \mathrm{kPa}, \operatorname{Emax}=128.1 \mathrm{kPa}$, Eratio =3.1. SWE, shear wave elastography; TNBC, triple negative breast cancer: Emean, mean; Emax, maximum; Emin, minimum; Eratio, ratio to normal gland; Red color, higher stiffness; Blue color, lower stiffness.

Table 2 Comparison of characteristics between TNBC and non-TNBC

\begin{tabular}{|c|c|c|c|c|c|c|}
\hline Group & Number & Emean (kPa) & $\operatorname{Emax}(\mathrm{kPa})$ & Emin (kPa) & $\mathrm{SD}(\mathrm{kPa})$ & Eratio \\
\hline Non-TNBC & 78 & $132.76 \pm 34.87$ & $163.76 \pm 45.87$ & $84.87 \pm 29.98$ & $13.31 \pm 8.22$ & $15.14 \pm 8.88$ \\
\hline$\chi^{2}$ & & -5.543 & -7.8875 & -1.99 & -1.198 & -5.117 \\
\hline$P$ & & 0.000 & 0.000 & 0.032 & 0.006 & 3.765 \\
\hline
\end{tabular}

TNBC, triple negative breast cancer; Emean, mean; Emax, maximum; Emin, minimum; SD, standard deviation; Eratio, ratio to normal gland.

liquidation, and hemorrhage. In addition, compared with non-TNBC lesions, internal micro-calcification was rare in TNBC lesions, which conformed to the research results by Wei et al. (26) and Ko et al. (27). However, some scholars have also found that the difference in micro-calcification between the 2 is not statistically significant, which may be related to the fact that US has poor visualization for microcalcification and poor detection repeatability due to the influence of micro-calcification size and density $(28,29)$.

Our research results suggested that the TIMI and resistance index of lesions in the TNBC group were similar to those in the non-TNBC group, and the differences were not statistically significant, which was consistent with previous research results. The possible reason may be that the formation of new blood vessels lays the foundation for blood flow formation within the lesion, which is not directly associated with the expression of ER, PR, and HER-2, instead, it is mainly affected by the vascular endothelial growth factor (VEGF) produced by malignant tumor cells.

In this research, the Emean, Emax, and Eratio values in the non-TNBC group were greater than those in the TNBC group, which was consistent with the research by Džoić Dominković et al. (30), which also suggested that SWE could provide a new technical means to distinguish the hardness difference between the 2 categories of BC. Chamming's et al. (31) discovered that the lesion hardness was positively correlated with fibrosis, while negatively correlated with necrosis, but it showed no correlation with cells and tissues. As mentioned previously, TNBC displayed "squeezing" growth, a large number of tumor cells had crowded together, the tumor cells were nest-like, and regional liquidation and necrosis were commonly seen due to insufficient blood supply. In contrast, the non-TNBC tumor cells showed "invasive" growth, accompanied by surrounding connective tissue responsive hyperplasia, and increased collagen fiber component; moreover, the high expression of estrogen and other cytokines could remarkably 


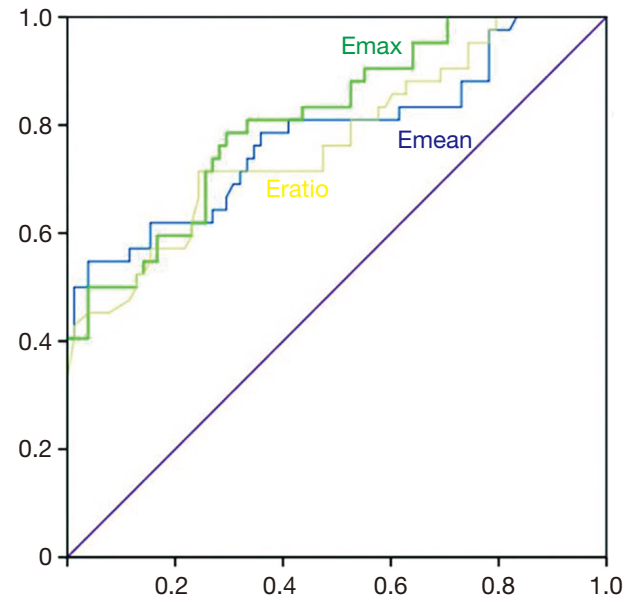

Figure 4 ROC curve of the diagnosed lesion. ROC, receiver operating characteristic.

promote the synthesis of fibroblasts and collagen (32), thus increasing the lesion hardness. Consequently, in this research, the Emean in the ROI was greater in the nonTNBC group than in the TNBC group. Kobayashi T (33) utilized the Emean ratio of lesion to fat to obtain the Eratio value. However, we believed that there was a great difference in the mammary gland density among different individuals due to the influences of age and hormone level, while the fat density was not greatly changed. Using the ratio of lesion to normal gland could maximally reduce the difference among different individuals, improve the accuracy and repeatability. Our research results suggested that the Eratio value in the TNBC group was smaller than that in the non-TNBC group. In addition, the inconsistent proliferation rate and complex composition of cancer cells had resulted in the incompletely uniform hardness within the ROI (33). In this research, Emax represented the tissue hardness at the hardest position within the ROI of the greatest hardness, thus, it could be speculated that that parameter might be the hardness value at the site with the most abundant mesenchymal composition and least tissue necrosis; as a result, the Emax in the non-TNBC group was greater than that in the TNBC group.

Nonetheless, there were several noteworthy limitations in this research: (I) factors such as individual difference, clinical TNM stage, histological grade, and special type BC had not been considered in the participants. However, tumor progression is a dynamic process, and there may be different changes in morphology, blood supply, and hardness at various stages, which should be further investigated in studies with a larger sample size. (II) This study was a small-sample-size retrospective study, and further multi-center and large sample-sized studies should be carried out to verify our findings.

In summary, TNBC mostly manifests as micro-lobular margin, with clear boundary, and no internal microcalcification; in comparison, non-TNBC mostly manifests as margin angulation or spur, with hyper echo halo in the boundary, accompanied by internal micro-calcification. It can be discovered using the SWE technique that, TNBC lesions have lower hardness than non-TNBC lesions.

In conclusion, conventional US combined with SWE can provide more imaging information for the early differential diagnosis of TNBC, and increase the diagnostic confidence in sonographers, which is a favorable prospect for clinical application.

\section{Acknowledgments}

Funding: This work was supported by Medical Scientific Research Foundation of Zhejiang Province, China (2019KY596) and Medical Scientific Research Foundation of Zhejiang Province, China (2020KY852).

\section{Footnote}

Reporting Checklist: The authors have completed the STARD reporting checklist. Available at https://dx.doi. org/10.21037/gs-21-320

Data Sharing Statement: Available at https://dx.doi. org/10.21037/gs-21-320

Conflicts of Interest: All authors have completed the ICMJE uniform disclosure form (available at https://dx.doi. org/10.21037/gs-21-320). The authors have no conflicts of interest to declare.

Ethical Statement: The authors are accountable for all aspects of the work in ensuring that questions related to the accuracy or integrity of any part of the work are appropriately investigated and resolved. All procedures performed in this study involving human participants were in accordance with the Declaration of Helsinki (as revised in 2013). This study was approved and supervised by the Research Ethics Committee of the Hwa Mei Hospital (NO.: YJ-KYSB-NBEY-2018-055-01). Informed consent was provided by all participants. 
Open Access Statement: This is an Open Access article distributed in accordance with the Creative Commons Attribution-NonCommercial-NoDerivs 4.0 International License (CC BY-NC-ND 4.0), which permits the noncommercial replication and distribution of the article with the strict proviso that no changes or edits are made and the original work is properly cited (including links to both the formal publication through the relevant DOI and the license). See: https://creativecommons.org/licenses/by-nc-nd/4.0/.

\section{References}

1. Fan L, Strasser-Weippl K, Li JJ, et al. Breast cancer in China. Lancet Oncol 2014;15:e279-89.

2. Oner G, Altintas S, Canturk Z, et al. Triple-negative breast cancer-Role of immunology: A systemic review. Breast J 2020;26:995-9.

3. Dent R, Trudeau M, Pritchard KI, et al. Triple-negative breast cancer: clinical features and patterns of recurrence. Clin Cancer Res 2007;13:4429-34.

4. Marra A, Viale G, Curigliano G. Recent advances in triple negative breast cancer: the immunotherapy era. BMC Med 2019;17:90.

5. McCann KE, Hurvitz SA, McAndrew N. Advances in Targeted Therapies for Triple-Negative Breast Cancer. Drugs 2019;79:1217-30.

6. Tan AR, Swain SM. Therapeutic strategies for triplenegative breast cancer. Cancer J 2008;14:343-51.

7. Goldhirsch A, Winer EP, Coates AS, et al. Personalizing the treatment of women with early breast cancer: highlights of the St Gallen International Expert Consensus on the Primary Therapy of Early Breast Cancer 2013. Ann Oncol 2013;24:2206-23.

8. Lehmann BD, Bauer JA, Chen X, et al. Identification of human triple-negative breast cancer subtypes and preclinical models for selection of targeted therapies. J Clin Invest 2011;121:2750-67.

9. Yang X, Zhang K, Zhang C, et al. Accuracy of analysis of cfDNA for detection of single nucleotide variants and copy number variants in breast cancer. BMC Cancer 2019;19:465.

10. Dogan BE, Turnbull LW. Imaging of triple-negative breast cancer. Ann Oncol 2012;23 Suppl 6:vi23-9.

11. Tian L, Wang L, Qin Y, et al. Systematic Review and Meta-analysis of the Malignant Ultrasound Features of Triple-Negative Breast Cancer. J Ultrasound Med 2020;39:2013-25.

12. Polat AV, Ozturk M, Polat AK, et al. Efficacy of
Ultrasound and Shear Wave Elastography for the Diagnosis of Breast Cancer-Related Lymphedema. J Ultrasound Med 2020;39:795-803.

13. Mercado CL. BI-RADS update. Radiol Clin North Am 2014;52:481-7.

14. Adler DD, Carson PL, Rubin JM, et al. Doppler ultrasound color flow imaging in the study of breast cancer: preliminary findings. Ultrasound Med Biol 1990;16:553-9.

15. Cleator S, Heller W, Coombes RC. Triple-negative breast cancer: therapeutic options. Lancet Oncol 2007;8:235-44.

16. Riva F, Bidard FC, Houy A, et al. Patient-Specific Circulating Tumor DNA Detection during Neoadjuvant Chemotherapy in Triple-Negative Breast Cancer. Clin Chem 2017;63:691-9.

17. Mukhopadhyay UK, Oturkar CC, Adams C, et al. TP53 Status as a Determinant of Pro- vs Anti-Tumorigenic Effects of Estrogen Receptor-Beta in Breast Cancer. J Natl Cancer Inst 2019;111:1202-15.

18. Tischkowitz M, Brunet JS, Bégin LR, et al. Use of immunohistochemical markers can refine prognosis in triple negative breast cancer. BMC Cancer 2007;7:134.

19. Zhong W, Chen S, Qin Y, et al. Doxycycline inhibits breast cancer EMT and metastasis through PAR1/NF- B/miR-17/E-cadherin pathway. Oncotarget 2017;8:104855-66.

20. Liu B, Fan Z, Edgerton SM, et al. Metformin induces unique biological and molecular responses in triple negative breast cancer cells. Cell Cycle 2009;8:2031-40.

21. Xi X, Liu N, Wang Q, et al. ACT001, a novel PAI-1 inhibitor, exerts synergistic effects in combination with cisplatin by inhibiting PI3K/AKT pathway in glioma. Cell Death Dis 2019;10:757.

22. Francies FZ, Herd O, Cairns A, et al. Chromosomal radiosensitivity of triple negative breast cancer patients. Int J Radiat Biol 2019;95:1507-16.

23. Zhao N, Peng F, Chen X. The Unfolded Protein Response in Triple-Negative Breast Cancer. The Gröbner Cover 2019.

24. Kojima Y, Tsunoda H. Mammography and ultrasound features of triple-negative breast cancer. Breast Cancer 2011;18:146-51.

25. Li B, Zhao X, Dai SC, et al. Associations between mammography and ultrasound imaging features and molecular characteristics of triple-negative breast cancer. Asian Pac J Cancer Prev 2014;15:3555-9.

26. Wei Y, Li Z, Zeng J. The Value of Ultrasonic Elastography and Conventional Ultrasonography in the Diagnosis 
of Triple-negative Breast Cancer. Chinese Journal of Ultrasound in Medicine 2012.

27. Ko ES, Lee BH, Kim HA, et al. Triple-negative breast cancer: correlation between imaging and pathological findings. Eur Radiol 2010;20:1111-7.

28. Huo HP, Huo JB, Wan WB, et al. Construction of Nude Mouse Model of Triple Negative Breast Cancer and Value of Thermal Tomography. Zhongguo Yi Xue Ke Xue Yuan Xue Bao 2016;38:198-204.

29. Zhong $W$, Yang $W$, Qin $Y$, et al. 6-Gingerol stabilized the p-VEGFR2/VE-cadherin/ $\beta$-catenin/actin complex promotes microvessel normalization and suppresses tumor progression. J Exp Clin Cancer Res 2019;38:285.

30. Džoić Dominković M, Ivanac G, Kelava T, et al.

Cite this article as: Sheng C, Gao S, Yan L, Yin H, Hu J, Ye Z, Wei X. Application value of conventional ultrasound combined with shear wave elastography in diagnosing triple negative breast cancer. Gland Surg 2021;10(6):1980-1988. doi: 10.21037 /gs-21-320
Elastographic features of triple negative breast cancers. Eur Radiol 2016;26:1090-7.

31. Chamming's F, Latorre-Ossa H, Le Frère-Belda MA, et al. Shear wave elastography of tumour growth in a human breast cancer model with pathological correlation. Eur Radiol 2013;23:2079-86.

32. Çebi Olgun D, Korkmazer B, Kılıç F, et al. Use of shear wave elastography to differentiate benign and malignant breast lesions. Diagn Interv Radiol 2014;20:239-44.

33. Kobayashi T. Review: ultrasonic diagnosis of breast cancer. Ultrasound Med Biol 1975;1:383-91.

(English Language Editor: J. Jones) 